\title{
Satiety and substrate mobilization after oral fat stimulation
}

\author{
Astrid J. P. G. Smeets* and Margriet S. Westerterp-Plantenga \\ Maastricht University, Department of Human Biology, PO Box 616, 6200 MD, Maastricht, The Netherlands, and Wageningen \\ Centre for Food Sciences, PO Box 557, 6700 AN, Wageningen, The Netherlands
}

(Received 1 July 2005 - Revised 8 December 2005 - Accepted 9 December 2005)

\begin{abstract}
The aim of the study was to provoke cephalic and metabolic responses by oral fat stimulation with different high-fat meals in the postprandial state. A randomized parallel design was executed with three groups of subjects (twenty-six women and ten men; twelve subjects per group). Oral fat stimulation was achieved by the modified sham feeding (MSF) technique. Five hours after a high-fat breakfast, the subjects were given one of three test meals in random order: a high-fat lunch, water or the same lunch as the MSF. The main fat sources in the high-fat lunch and MSF were olive oil, linoleic oil and oleic oil. For $3 \mathrm{~h}$ after the test meal, blood samples were taken for analysis of metabolite, and visual analogue scales of appetite profile were completed. A cephalic response appeared to be achieved by MSF in that we observed a relative increase in insulin and glucose; this response lasted up until $90 \mathrm{~min}$, indicating possible vagal stimulation. NEFA increased significantly after MSF compared with water ingestion in the case of olive oil $(P<0 \cdot 0001)$ and linoleic oil $(P<0 \cdot 05)$, but not with oleic oil. MSF provoked a significantly higher increase in triacylglycerol and glycerol levels compared with water ingestion in the case of linoleic oil $(P<0 \cdot 05)$. Satiety was significantly increased in the eating condition and in the MSF condition $(P<0.0002$, all oils $)$ compared with water ingestion. We conclude that cephalic and perhaps vagal stimulation by different fats increased the concentrations of the metabolites and stimulated satiety, with linoleic oil showing the strongest response.
\end{abstract}

Fat perception: Cephalic response: Satiety

Obesity and related disorders such as the metabolic syndrome are rapidly growing nutrition-related diseases worldwide (James et al. 2004). Metabolic targets for improving bodyweight management are, among others, satiety and substrate metabolism. With regard to satiety, it is interesting to see that cephalic, enteric and intestinal phase responses follow nutrient intake. Some of these reactions may even start before the actual ingestion of food, whereas other reactions may last for hours in the postprandial period (Rasmussen et al. 1990; Strubbe, 1992; Konturek et al. 2003). The thought, sight, smell and taste of food may initiate cephalic responses, which influence the absorption and utilization of nutrients through vagal cholinergic activity (Naim et al. 1978; Hopman et al. 1987). Vagal responses may have long-lasting effects on metabolism as they have been suggested to be involved in the improvement of postprandial glucose tolerance and satiety and the elevation of postprandial lipaemia after a gastric fat load (Robertson et al. 2001).

Until now, few studies have examined the effects of the oral stimuli that occur before and during food ingestion, especially fat ingestion, on postprandial or postabsorptive metabolism. High-fat foods are not simply rich in energy, but are also perceived as more palatable in general (Drewnowski, 1995, 1997). Important indicators of whether fat is present in a food are texture and odour (Rolls et al. 1999). Schemmel and co-workers observed a 'taste for fat' in specific rat strains by discovering an inverse relationship between the perception of NEFA and dietary preferences for fat. When placed on a high-fat diet, fat-preferring rats became obese whereas fatavoiding rats stayed lean (Schemmel et al. 1970).

Further investigations by Gilbertson and co-workers revealed a dose-dependent detection of low concentrations of NEFA (linoleic acid) by an inhibition of delayed rectifying $\mathrm{K}^{+}$channels in the taste receptor cells of rats, causing an increase in activity of these cells (Gilbertson et al. 1997). Human subjects are able to detect linoleic acid in these low concentrations as well (Kamphuis et al. 2003). Experiments using oral stimulation may shed some light on the consequences of vagal stimulation with fat.

Previous oral stimulation experiments using the modified sham feeding (MSF) technique have revealed effects on metabolites, hormones and satiety. Mattes and co-workers (Mattes, 1996, 2001a,b, 2002; Tittelbach \& Mattes, 2001, 2002) found that oral stimulation after a gastric fat load resulted in a higher plasma triacylglycerol level. Robertson's group (Robertson et al. 1999, 2000, 2002; Jackson et al. 2000 , 2001) performed oral stimulation experiments in the postprandial phase in which they observed a sustained suppression of plasma NEFA. In the experiments of Heath et al. (2004), oral stimulation $1 \mathrm{~h}$ before a fat load resulted in a further decline of ghrelin levels, which was interpreted as a decrease in hunger. 
The aim of the present study was to further assess cephalic and metabolic responses using vagal stimulation in the postprandial state. We investigated cephalic and metabolic responses (i.e. metabolites and satiety) to oral stimulation using three different high-fat meals, which were rich in one of three oils: (1) olive oil, (2) an oil rich in linoleic acid or (iii) an oil rich in oleic acid, in the postprandial phase. The design consisted of a high-fat meal following a high-fat breakfast. Previous oral stimulation experiments have shown effects of MSF using high-fat meals in the postprandial period (Robertson et al. 2001, 2002). In contrast to the MSF meals in previous studies, the MSF meals in the current study contained very little carbohydrate and protein, which allows us to look particularly at the effects of fat. The three different oils (olive oil, linoleic acid and oleic acid) were used to assess the effects of MUFA and PUFA. Olive oil was chosen because of its possible effects on triacylglycerol-rich chylomicron particles (Jackson et al. 2002), and linoleic acid because it is believed that it can be specifically perceived, with oleic acid as its control (Schemmel et al. 1970; Gilbertson et al. 1997).

\section{Subjects and methods}

\section{Subjects}

Thirty-six subjects (twenty-six women: BMI 22.4 (SD 1.8) kg/ $\mathrm{m}^{2}$, age 29.9 (SD 13.6) $\mathrm{kg} / \mathrm{m}^{2}$ years; ten men: BMI 23.1 (SD $2.0) \mathrm{kg} / \mathrm{m}^{2}$, age 30.6 (SD 12.6) $\mathrm{kg} / \mathrm{m}^{2}$ years; twelve subjects per group) were studied on three occasions, in a series of experiments with one type of fat. The subjects were divided into three treatment groups (Table 1). The subjects were selected on the basis of age, height, weight, BMI and restrained eating score on the Three Factor Eating Questionnaire (Stunkard \& Messick, 1985). All subjects were healthy, not taking medication, non-smokers and not dieting. All subjects gave written informed consent, and the study was approved by the Maastricht University Ethics Committee.

\section{Study protocol}

A randomized parallel design was executed with three groups. On the day before each study day, subjects consumed at home a low-fat evening meal ( $15 \%$ energy derived from fat), which was provided by us. Before breakfast on the test day, the subjects were asked whether they had consumed the low-fat evening meal the previous evening. On each test day, subjects consumed breakfast at 08.00 hours; this consisted of two croissants with butter and Brie cheese, and a cup of chocolate milk. Five hours after the high-fat breakfast, the subjects were given

Table 1. Characteristics of the subjects

(Means and their standard deviations)

\begin{tabular}{|c|c|c|c|c|c|c|}
\hline & \multicolumn{2}{|c|}{ Olive oil } & \multicolumn{2}{|c|}{ Oleic } & \multicolumn{2}{|c|}{ Linoleic } \\
\hline & Mean & SD & Mean & SD & Mean & SD \\
\hline$n$ & 12 & & 12 & & 12 & \\
\hline Women & 8 & & 9 & & 9 & \\
\hline Men & 4 & & 3 & & 3 & \\
\hline Age & $44 \cdot 3$ & $10 \cdot 8$ & $23 \cdot 1$ & $7 \cdot 1$ & $22 \cdot 8$ & $6 \cdot 8$ \\
\hline BMI $\left(\mathrm{kg} / \mathrm{m}^{2}\right)$ & $23 \cdot 6$ & $2 \cdot 4$ & 22.6 & $1 \cdot 3$ & $21 \cdot 7$ & $1 \cdot 1$ \\
\hline
\end{tabular}

one of three test meals in randomized order: a high-fat lunch, water or the same lunch as MSF.

The macronutrient compositions of the meals are specified in Table 2. The main fat sources of the lunch in the three different treatments were olive oil, an oil rich in linoleic acid (Becel; Unilever, Vlaardingen, The Netherlands) and an oil rich in oleic acid (Hozol oil; Contined BV, Bennekom, The Netherlands), respectively. For the olive oil treatment, the lunch consisted of a salad and whipped cream with orange-flavoured energy-free lemonade syrup (Cereal Diaran; Novartis Consumer Health BV, Breda, The Netherlands). In the olive oil treatment group, we used whipped cream because we expected the cream would give an optimal oral fat exposure. In the linoleic acid and oleic acid treatment groups, the lunch consisted of a soup and a salad, in order to simplify the manipulation with the type of fat. Blood samples were taken from the antecubital vein just before and 15, 30, 60, 90 and $120 \mathrm{~min}$ after the lunch.

At the start of the lunch meal, subjects were asked to fill in $100 \mathrm{~mm}$ visual analogue scales (VAS) on their feelings of hunger, fullness, appetite, satiety, thirst, prospective food consumption, desire to eat and palatability of each course. Additionally, subjects were asked to fill in $100 \mathrm{~mm}$ VAS at six different time points after the lunch on their feelings of hunger, fullness, appetite, satiety, thirst, prospective food consumption and desire to eat. The scale ranged from 'not at all' on the left to 'extremely' on the right.

\section{Modified sham feeding}

In the MSF condition, the subjects were presented with the test meal and were instructed to chew the food until the point at which they would normally swallow and then to expectorate the food into a plastic cup. They were continually instructed not to swallow any food. The subjects repeated the procedure until the meal had been fed completely (15-20 min). The weights of each meal were measured before and after MSF.

\section{Blood collection and analytical methods}

Blood samples were collected in tubes containing EDTA to prevent clotting. Plasma was obtained by centrifugation $\left(4^{\circ} \mathrm{C}, 3000 \mathrm{rpm}, 10 \mathrm{~min}\right)$ and stored at $-20^{\circ} \mathrm{C}$ until analysis of glucose by a hexokinase method (ABX Diagnostics, Montpellier, France), insulin (RIA kit; Linco Research, St Charles, MO, USA) NEFA (NEFA C-kit Wako 994-75409; Sopar Biochemicals, Koekelberg, Belgium), triacylglycerol (GPO-trinder 337; Sigma Diagnostics Inc., St Louis, MO, USA) and glycerol by a glycerolkinase-lipase method (Boehringer, Mannheim, Germany) using a semi-automated

Table 2. Composition of the meals

\begin{tabular}{lccccc}
\hline & $\begin{array}{c}\text { Weight } \\
(\mathrm{g})\end{array}$ & $\begin{array}{c}\text { Energy } \\
(\mathrm{kJ})\end{array}$ & $\begin{array}{c}\text { Protein } \\
(\mathrm{g})\end{array}$ & $\begin{array}{c}\text { Fat } \\
(\mathrm{g})\end{array}$ & $\begin{array}{c}\text { Carbohydrates } \\
(\mathrm{g})\end{array}$ \\
\hline Evening meal & 475 & 2290 & 19 & 9.5 & 95 \\
Breakfast & 355 & 3656 & 23.3 & 59.4 & 61.35 \\
Lunch olive oil & 285 & 1446.3 & 2.9 & 35.7 & 4.3 \\
Lunch oleic & 443 & 1461.3 & 3.7 & 33.0 & 9.8 \\
Lunch linoleic & 443 & 1461.3 & 3.7 & 33.0 & 9.8 \\
\hline
\end{tabular}


centrifugal spectrophometer (Cobas Fara; Roche Diagnostics Basel, Switzerland).

\section{Statistical analysis}

All data are presented as means and standard deviations or means with their standard errors. Areas under the curve (AUC) of the percentage change in concentration over 120 min were calculated using the trapezoid method. The three conditions (water, MSF and eating) were compared within each treatment group (olive oil, oil rich in linoleic acid and oil rich in oleic acid) in terms of the postprandial metabolite responses, hormone responses and VAS scores by using repeated-measures ANOVA. Differences between the oleic acid treatment group and the linoleic acid treatment group in terms of the postprandial metabolite responses, hormone responses and VAS scores were analysed using factorial ANOVA. Post hoc analysis was carried out with a Scheffe F-test or a Fisher PLSD test. All of the statistical analyses were made with Statview SE Graphics software (version 4.5; Abacus Concepts Inc, Berkeley, CA, USA), and the criterion for significance was set at $P<0 \cdot 05$.

\section{Results}

Subjects

The subjects in the three groups were all selected based on the same criteria. The subjects in the olive oil group were, however, significantly $(P<0.0001)$ older than those in the two other groups.

\section{Palatability of the lunches}

The subjects rated their lunch, which was completely new to them, as sufficiently and similarly palatable (olive oil treatment group 5.9 (SEM 1.6) $\mathrm{cm}$; linoleic acid treatment group 6.2 $($ SEM 1.7$) \mathrm{cm}$; oleic acid treatment group $6.3($ SEM $1 \cdot 7) \mathrm{cm}$ ).

\section{Expectorated meals}

The weights of the MSF meal before and after chewing were compared; this yielded a mean recovery rate of 99.8 (SEM $2.58) \%$. The recovery rates from the MSF meal in the olive oil, linoleic acid oil and oleic acid oil groups were not significantly different.

\section{Blood samples}

An overview of the results of the blood analyses calculated as AUC delta $\%$ is given in Table 3.

Glucose. We observed an increase in glucose concentration with oleic acid over the period 15-90 min (calculated as AUC delta \%) after MSF and after eating compared with the water condition $(P<0.0001 ;$ Fig. 1$)$. In the olive oil and linoleic acid treatment groups, we observed a significant increase in glucose concentrations after eating compared with the MSF and water conditions, but no significant difference between the MSF and water conditions $(P<0 \cdot 001$; data not shown).

Insulin. Insulin concentrations (calculated as AUC delta \%) increased significantly more after MSF compared

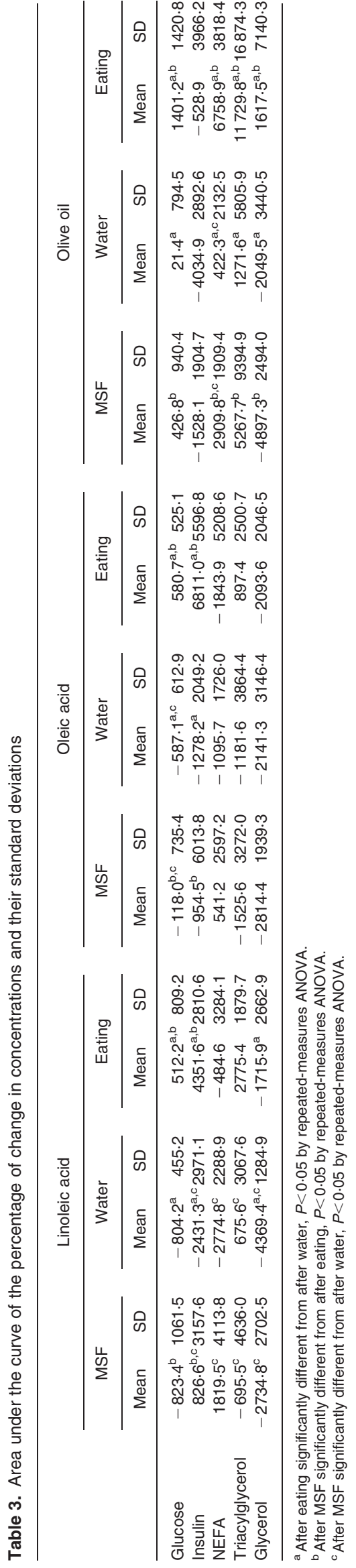




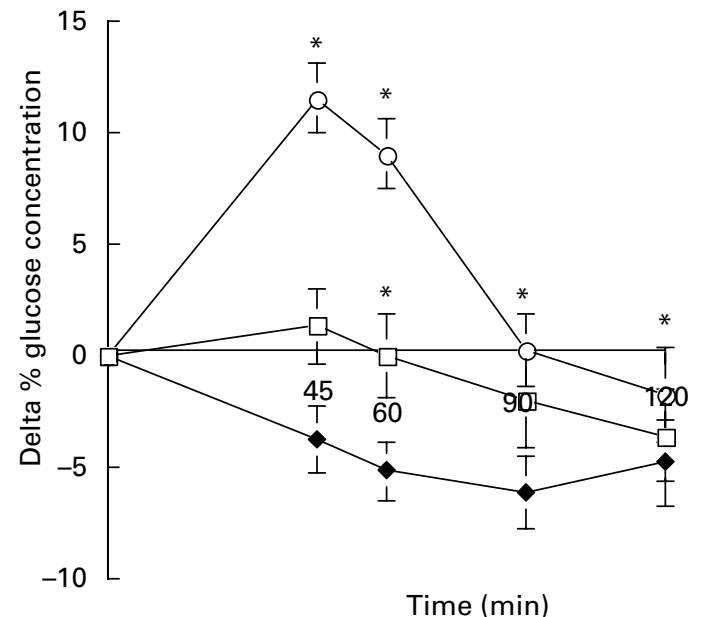

Fig. 1. Mean and SEM percentage of change in plasma glucose concentration with the oleic acid treatment. *After eating or MSF significantly different from after water, $P<0.05$ by repeated-measures ANOVA. $-\square-\mathrm{MSF}$, oleic; $\longrightarrow$ - water, oleic; $-\mathrm{O}-$ eating, oleic.

with the water condition, and after eating compared with the water condition in the case of linoleic acid $(P<0 \cdot 0001$; Fig. 2). In the oleic acid treatment group, insulin concentrations increased significantly more after eating compared with the MSF and water conditions $(P<0.0004)$. We observed no significant differences in insulin concentrations after the MSF condition compared with the water condition in the oleic acid treatment group. Insulin concentrations after all conditions (MSF, eating and water) were not significantly different in the olive oil treatment group (data not shown).

Non-esterified fatty acids. NEFA increased significantly (calculated as AUC delta \%) after MSF compared with the water condition, and after eating compared with the water condition, in the case of olive oil $(P<0 \cdot 0001$; Fig. 3). NEFA increased significantly after MSF compared with the water condition in the case of linoleic acid $(P<0.05$; Fig. 4). NEFA concentrations after all conditions (MSF, eating and water) were not significantly different in the oleic acid treatment group (data not shown).

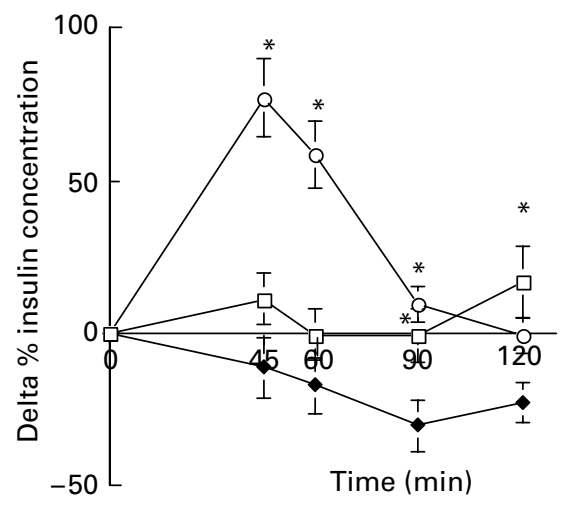

Fig. 2. Mean and SEM percentage of change in plasma insulin concentration with the linoleic acid treatment. *After eating or MSF significantly different from after water, $P<0.05$ by repeated-measures ANOVA. $-\square-$ MSF, linoleic; $\longrightarrow-$ water, linoleic; —O— eating, linoleic.

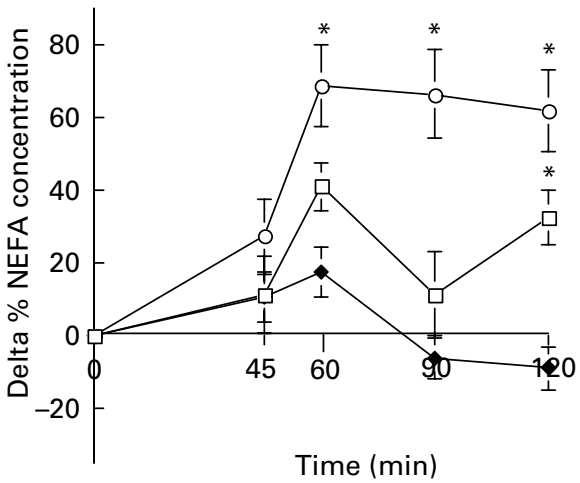

Fig. 3. Mean and SEM percentage of change in plasma NEFA concentrations with the olive oil treatment. *After eating or MSF significantly different from after water, $P<0.05$ by repeated-measures ANOVA. - $\square-$ MSF, olive oil; $\longrightarrow$ - water, olive oil; —O— eating, olive oil.

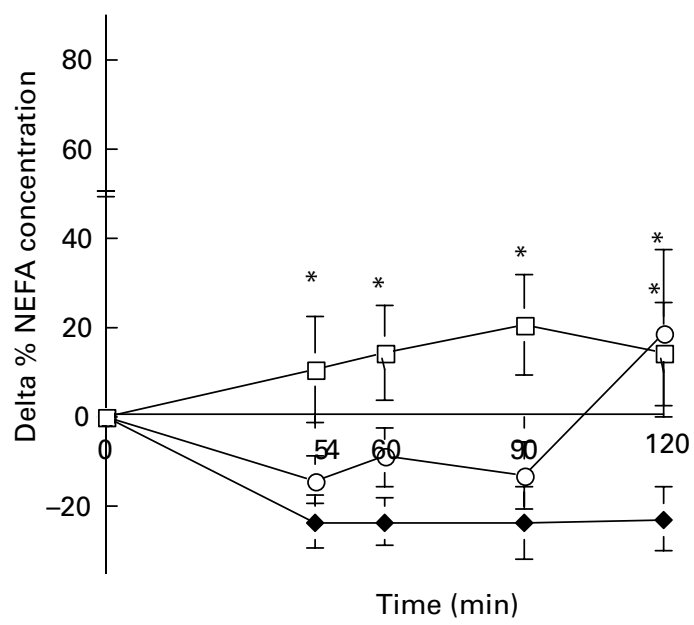

Fig. 4. Mean and SEM percentage of change in plasma NEFA concentrations with the linoleic acid treatment. *After eating or MSF significantly different from after water, $P<0.05$ by repeated-measures ANOVA. $-\square-$ MSF, linoleic; $\longrightarrow \longrightarrow$ water, linoleic; —O— eating, linoleic.

Triacylglycerol and glycerol. MSF and eating provoked a significantly higher increase in triacylglycerol concentrations (calculated as AUC delta \%) compared with the water condition in the case of linoleic acid $(P<0 \cdot 05$; Fig. 5). The triacylglycerol concentrations following the eating condition were significantly higher than the triacylglycerol concentrations following the MSF and water condition in the olive oil treatment group. In the oleic acid treatment group, we observed no significant difference in triacylglycerol concentrations after all conditions (data not shown). Additionally, the increase in triacylglycerol concentrations following MSF in the linoleic acid treatment group was significantly different from the decline in triacylglycerol concentration following MSF in the oleic acid treatment group $(P<0 \cdot 01)$.

Glycerol concentrations (calculated as AUC delta \%) increased significantly after MSF compared with the water condition, in the case of linoleic acid $(P<0 \cdot 05 ;$ Fig. 6$)$. The glycerol 


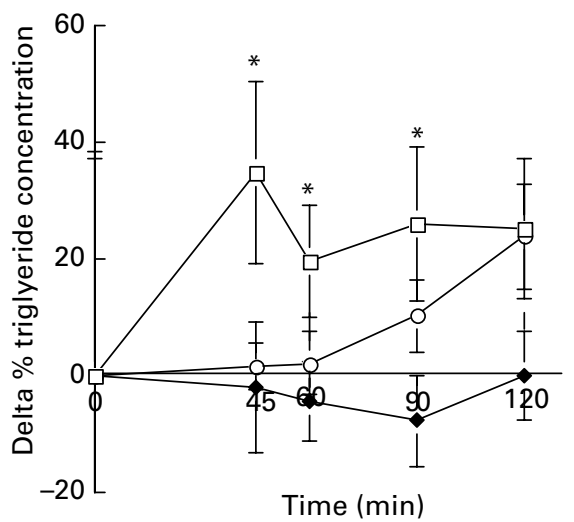

Fig. 5. Mean and SEM percentage of change in plasma triacylglycerol concentration with the linoleic acid treatment. *After eating or MSF significantly different from after water, $P<0.05$ by repeated-measures ANOVA. - $\square-$ MSF, linoleic; $\longrightarrow-$ water, linoleic; $-\mathrm{O}-$ eating, linoleic.

concentrations following the eating condition were significantly different from the glycerol concentrations following the MSF condition in the olive oil treatment group. In the oleic acid treatment group, we observed no significant difference in glycerol concentrations after all conditions (data not shown).

\section{Appetite profile}

Satiety (calculated as AUC delta) was not only significantly increased in the eating condition $(P<0.0002$, for all oils), but also, albeit to a smaller extent, in the MSF condition $(P<0.0002$, for all oils) compared with the water condition (Fig. 7).

Feelings of fullness (calculated as AUC delta) were significantly increased in the eating condition $(P<0 \cdot 0001$, for all oils) and in the MSF condition compared with the water condition in the linoleic acid $(P<0 \cdot 0001)$ and oleic acid $(P<0.0001)$ treatment groups. Hunger, appetite and desire to eat (calculated as AUC delta) were significantly increased in the eating condition $(P<0 \cdot 001$, for all oils) and in the MSF condition compared with the water condition in the linoleic acid $(P<0.001)$ and oleic acid $(P<0.001)$ treatment groups (data not shown).

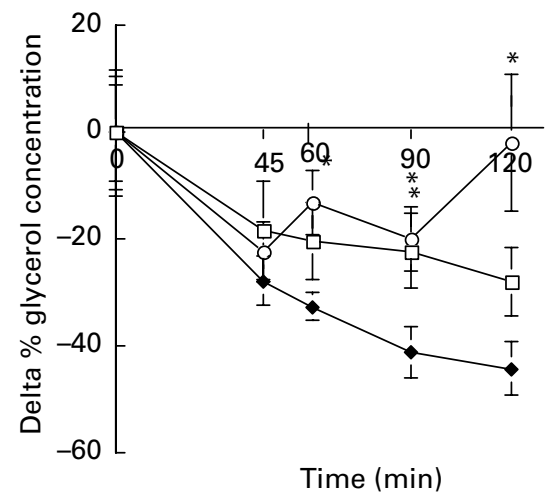

Fig. 6. Mean and SEM percentage of change in plasma glycerol concentration with the linoleic acid treatment. *After eating or MSF significantly different from after water, $P<0.05$ by repeated-measures ANOVA. $-\square-\mathrm{MSF}$, linoleic; $\longrightarrow \longrightarrow$ water, linoleic; —O— eating, linoleic.
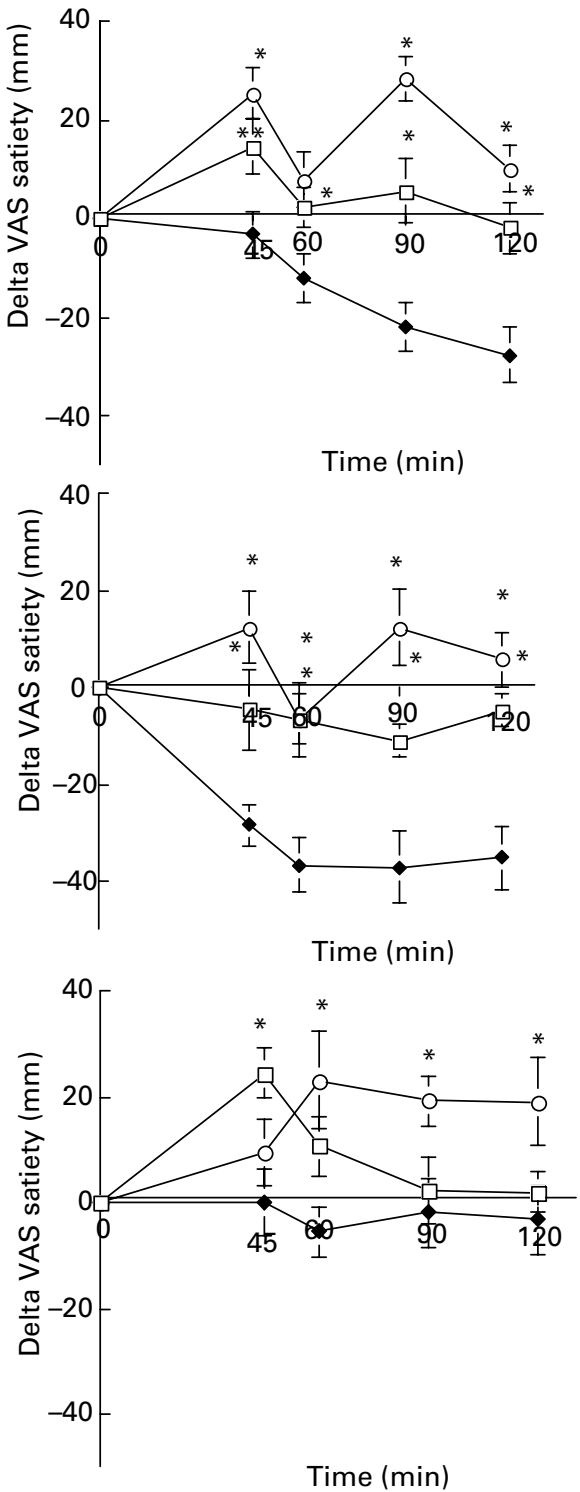

Fig. 7. Mean and SEM change in feelings of satiety $(\mathrm{mm})$ with the (a) linoleic acid, (b) oleic acid and (c) olive oil treatment. *After eating or MSF significantly different from after water, $P<0.0002$ by repeated-measures ANOVA. $\longrightarrow \square-\mathrm{MSF} ; \longrightarrow-$ water; $\longrightarrow \mathrm{O}-$ eating.

\section{Discussion}

The oral fat stimulation by MSF of different fats, i.e. olive oil and linoleic oil, appeared to increase the release of triacylglycerol or NEFA. Moreover, feelings of satiety were increased with MSF of all oils, compared with water ingestion. This short increase in rated satiety following MSF is clearly a sensory effect, as absorptive effects are absent (Blundell et al. 1988). These effects on feelings of satiety following MSF may be mediated by satiety-related metabolites and hormones. Heath and co-workers observed increased feelings of fullness over $5 \mathrm{~h}$ when a meal was preceded by MSF $1 \mathrm{~h}$ earlier. This effect was accompanied by a sustained decline in plasma ghrelin concentration following MSF (Heath et al. 2004). These findings suggest that increased oral exposure may enhance satiety. 
The duration of the effects we observed as increased glucose and insulin concentrations indicate that not just a cephalic response, but also vagal stimulation appeared to be achieved by MSF (Ahren \& Holst, 2001). Similar effects on plasma glucose and insulin concentration following MSF with saturated fat have been observed by Robertson et al. (2001). It is not likely that the effects we observed in plasma concentrations following MSF are produced by the ingestion of food, as shown by the high total recovery of the weights of the expectorated meals. Earlier oral stimulation studies have shown that the amount of food that is accidentally swallowed during MSF is only a few grams (Mattes, 1996, 2001a, 2002; Jackson et al. 2000, 2001; Tittelbach \& Mattes, 2001; Robertson et al. 2002; Heath et al. 2004).

In two treatments, i.e. with olive oil and linoleic acid, we found that NEFA increased significantly after MSF compared with the water condition (Figs. 3 and 4). With these treatments, oral stimulation seems to increase the availability of oxidizable metabolites, without the subject receiving any exogenous nutrients. Previous studies using the MSF technique observed a sustained decline in plasma NEFA concentration (Robertson et al. 1999, 2001; Heath et al. 2004). The differences in the macronutrient compositions and fat sources of the meals between our study and previous studies may account for the difference in NEFA response. The meals we used in our study contained less carbohydrates and more fat; this may have affected the regulation of NEFA concentrations indirectly through effects on insulin or glucagon, which are both involved in lipolysis (Perea et al. 1995; Evans et al. 1999).

MSF provoked a significantly higher increase in triacylglycerol compared with the water condition only with the linoleic acid treatment. Because we observed this effect with the linoleic acid treatment, and to a smaller extent with the olive oil treatment, but not with the oleic acid treatment, oral stimulation with specific fatty acids may be required to induce a rise in NEFA and triacylglycerol. Additionally, the individual ability to detect specific fatty acids, in this case oleic acid and linoleic acid, might play a role in the observed effects. A study performed by Kamphuis \& Westerterp-Plantenga (MM Kamphuis and MS Westerterp-Plantenga, unpublished results) has shown that low concentrations of linoleic acid were detected by a higher percentage of the subjects than the same low concentrations of oleic acid. Thus, if a specific fatty acid is not detected, it cannot provoke a response, as may be the case in this experiment with oleic acid.

Postprandial studies in which sequential meals were used show that when a second meal is ingested $4-6 \mathrm{~h}$ after the first meal, there is a rapid appearance of chylomicrons in the circulation, with a peak $1 \mathrm{~h}$ after the second meal. These chylomicrons have been shown to carry fat ingested in the first meal (Peel et al. 1993; Fielding et al. 1996; Evans et al. 1998). It has been suggested that a proportion of fat from the first meal remains in the gut lumen or in the enterocyte and enters the plasma pool after ingesting a second meal (Jackson et al. 2002). Jackson et al. (2001) and Robertson et al. (2001) observed no elevation of plasma triacylglycerol, triacylglycerol-rich lipoprotein or triacylglycerol-rich lipoprotein-retinyl ester concentrations when MSF with either a low-fat or a moderate-fat meal was performed $5 \mathrm{~h}$ after a high-fat breakfast. In a different study, Jackson et al.
(2002) observed that the ingestion of a high-fat breakfast produced a rapid peak in triacylglycerol, retinyl esters and apo B48 chylomicrons after a second meal that was very low $(6 \mathrm{~g})$ in fat. These findings suggest that fat from the first meal is released in preformed chylomicrons and that the observed effects were not attributable to the second meal.

Further evidence for this 'storage theory' is supported by the findings of Mattes' group. They observed a release of fats from the previous (evening) meal following MSF with fat in combination with a fat load the next morning (Mattes, 2002). Previous experiments performed by Mattes and coworkers. focused on lipid metabolism using MSF with different dietary fats in combination with a fat load. The observed effects on lipid metabolism from these studies imply a taste component of dietary fats (Mattes, 1996, 2001a,b, 2002; Tittelbach \& Mattes, 2001). More recent experiments have observed increased plasma triacylglycerol concentrations when MSF was performed $1 \mathrm{~h}$ before a meal (Robertson et al. 2002; Heath et al. 2004).

In summary, it appears that the metabolic effects observed after the consumption of high-fat foodstuffs is at least partly influenced by the oral exposure to fats and the preceding meal. However, the mechanisms behind these effects still need to be clarified.

Because the subjects in the olive oil treatment group were significantly older than those in the oleic acid and the linoleic acid treatment groups, a comparison of the results of the olive oil treatment group with those of the other groups is not completely justified. Age-related changes in body composition and metabolism may have contributed to the absence of significant differences in insulin concentration across the three conditions (water, MSF and eating) in the olive oil group, and the higher responses (expressed as \% change) in NEFA and triacylglycerol in the olive oil group compared with the other two groups, as they are thought to contribute to a deterioration in insulin action and blood lipid profile (Kissebah et al. 1976; Kohrt et al. 1993; Vessby et al. 1994; Kelley et al. 1999; Kelley, 2002).

The significance of MSF with fats lies in the possible relationship between sensory perception and metabolic effects. Here, we showed that simply perception, without swallowing, evoked the appearance of metabolites and satiety. The increase in satiety was probably due to the increase in metabolites and hormones concomitant with the development of satiety (Woods, 2004). This study not only confirmed the observations reported by Robertson's group (Robertson et al. 1999, 2000, 2001, 2002; Jackson et al. 2000, 2001; Heath et al. 2004) and that of Mattes (Mattes, 1996, 2001a,b, 2002; Tittelbach \& Mattes, 2001, 2002), but also extended their findings to other fats, i.e. oil rich in linoleic acid, oil rich in oleic acid and olive oil, showing differences that depended on the type of fat and, moreover, showing effects on satiety. Further experiments should shed light on hormones involved in satiety to assess the mechanism of satiety and possible effects on substrate oxidation and thermogenesis.

\section{Acknowledgements}

We would like to thank Ilse Nijs, Joan Senden, Jos Stegen, Wendy Sluijsmans and Chantalle Moors for their contributions to the study. 


\section{References}

Ahren B \& Holst JJ (2001) The cephalic insulin response to meal ingestion in humans is dependent on both cholinergic and noncholinergic mechanisms and is important for postprandial glycemia. Diabetes 50(5), 1030-1038.

Blundell JE, Hill AJ \& Rogers PJ (1988) Hunger and the satiety cascade - their importance for food acceptance in the late 20th century. In Food acceptability, pp. 233-250 [DMH Thompson, editor]. Amsterdam: Elsevier Applied Science.

Drewnowski A (1995) Energy intake and sensory properties of food. Am J Clin Nutr 62, 1081S-1085S.

Drewnowski A (1997) Taste preference and food intake. Ann Rev Nutr 17, 237-253.

Evans K, Clark ML \& Frayn KN (1999) Effects of an oral and intravenous fat load on adipose tissue and forearm lipid metabolism. Am J Physiol 276, E241-E248.

Evans K, Kuusela PJ, Cruz ML, Wilhelmova I, Fielding BA \& Frayn KN (1998) Rapid chylomicron appearance following sequential meals: effects of second meal composition. $\mathrm{Br} J$ Nutr 79, 425-429.

Fielding BA, Callow J, Owen RM, Samra JS, Matthews DR \& Frayn KN (1996) Postprandial lipemia: the origin of an early peak studied by specific dietary fatty acid intake during sequential meals. Am J Clin Nutr 63, 36-41.

Gilbertson TA, Fontenot DT, Liu L, Zhang H \& Monroe WT (1997) Fatty acid modulation of $\mathrm{K}+$ channels in taste receptor cells: gustatory cues for dietary fat. Am J Physiol 272, C1203-C1210.

Heath RB, Jones R, Frayn KN \& Robertson MD (2004) Vagal stimulation exaggerates the inhibitory ghrelin response to oral fat in humans. J Endocrinol 180, 273-281.

Hopman WP, Jansen JB, Rosenbusch G \& Lamers CB (1987) Cephalic stimulation of gallbladder contraction in humans: role of cholecystokinin and the cholinergic system. Digestion 38, 197-203.

Jackson KG, Robertson MD, Deane LO, Fielding BA, Frayn KN \& Williams CM (2000) The effect of modified sham-feeding meals of varying fat content on postprandial triacylglycerol, insulin and glucose response. Proc Nutr Soc 59, 14A.

Jackson KG, Robertson MD, Fielding BA, Frayn KN \& Williams CM (2001) Second meal effects: modified sham feeding does not provoke the release of stored triacylglycerol from a previous highfat meal. Br J Nutr 85, 149-156.

Jackson KG, Robertson MD, Fielding BA, Frayn KN \& Williams CM (2002) Olive oil increases of triacylglycerol-rich chylomicron particles compared with other oils: an effect retained when a second meal is fed. Am J Clin Nutr 76, 942-949.

James PT, Rigby N \& Leach R (2004) The obesity epidemic, metabolic syndrome and future prevention strategies. Eur J Cardiovasc Prev Rehabil 11, 3-8.

Kamphuis MM, Saris WH \& Westerterp-Plantenga MS (2003) The effect of addition of linoleic acid on food intake regulation in linoleic acid tasters and linoleic acid non-tasters. $B r \quad J$ Nutr 90, 199-206.

Kelley DE (2002) Skeletal muscle triglycerides: an aspect of regional adiposity and insulin resistance. Ann N Y Acad Sci 967, $135-145$.

Kelley DE, Goodpaster B, Wing RR \& Simoneau JA (1999) Skeletal muscle fatty acid metabolism in association with insulin resistance, obesity, and weight loss. Am $J$ Physiol 277, E1130-E1141.

Kissebah AH, Alfarsi S, Adams PW \& Wynn V (1976) Role of insulin resistance in adipose tissue and liver in the pathogenesis of endogenous hypertriglyceridaemia in man. Diabetologia 12, $563-571$
Kohrt WM, Kirwan JP, Staten MA, Bourey RE, King DS \& Holloszy JO (1993) Insulin resistance in aging is related to abdominal obesity. Diabetes 42, 273-281.

Konturek SJ, Pepera J, Zabielski K, Konturek PC, Pawlik T, Szlachcic A \& Hahn EG (2003) Brain-gut axis in pancreatic secretion and appetite control. J Physiol Pharmacol 54, 293-317.

Mattes RD (1996) Oral fat exposure alters postprandial lipid metabolism in humans. Am J Clin Nutr 63, 911-917.

Mattes RD (2001a) Oral exposure to butter, but not fat replacers elevates postprandial triacylglycerol concentration in humans. J Nutr 131, 1491-1496.

Mattes RD (2001b) The taste of fat elevates postprandial triacylglycerol. Physiol Behav 74, 343-348.

Mattes RD (2002) Oral fat exposure increases the first phase triacylglycerol concentration due to release of stored lipid in humans. J Nutr 132, 3656-3662.

Naim M, Kare MR \& Merrie AM (1978) Effects of oral stimulation on the cephalic phase of pancreatic exocrine in dogs. Physiol Behav 20, 563-570.

Peel AS, Zampelas A, Williams CM \& Gould BJ (1993) A novel antiserum specific to apolipoprotein B-48: application in the investigation of postprandial lipidaemia in humans. Clin Sci (Lond) 85, $521-524$.

Perea A, Clemente F, Martinell J, Villanueva-Penacarrillo ML \& Valverde I (1995) Physiological effect of glucagon in human isolated adipocytes. Horm Metab Res 27, 372-375.

Rasmussen H, Zawalich KC, Ganesan S, Calle R \& Zawalich WS (1990) Physiology and pathophysiology of insulin secretion. Diabetes Care 13, 655-666.

Robertson MD, Jackson KG, Fielding BA, Williams CM \& Frayn KN (1999) Modified sham feeding of a modest-fat meal suppresses plasma non-esterified fatty acids. Proc Nutr Soc 59, 123A.

Robertson MD, Jackson KG, Williams CM, Fielding BA \& Frayn KN (2001) Prolonged effects of modified sham feeding on energy substrate mobilization. Am J Clin Nutr 73, 111-117.

Robertson MD, Mason AO \& Frayn KN (2000) Oro-sensory stimulation prior to fat ingestion impairs postprandial fat tolerance. Int $J$ Obesity 24, S55.

Robertson MD, Mason AO \& Frayn KN (2002) Timing of vagal stimulation affects postprandial lipid metabolism in humans. $\mathrm{Am}$ J Clin Nutr 76, 71-77.

Rolls ET, Critchley HD, Browning AS, Hernadi I \& Lenard L (1999) Responses to the sensory properties of fat of neurons in the primate orbitofrontal cortex. J Neurosci 19, 1532-1540.

Schemmel R, Mickelsen O \& Gill JL (1970) Dietary obesity in rats: body weight and body fat accretion in seven strains of rats. $J$ Nutr 100, 1041-1048.

Strubbe JH (1992) Parasympathetic involvement in rapid meal-associated conditioned insulin secretion in the rat. Am J Physiol 263, R615-R618.

Stunkard AJ \& Messick S (1985) The three-factor eating questionnaire to measure dietary restraint, disinhibition and hunger. $J$ Psychosom Res 29, 71-83.

Tittelbach TJ \& Mattes RD (2001) Oral stimulation influences postprandial triacylglycerol concentrations in humans: nutrient specificity. J Am Coll Nutr 20, 485-493.

Tittelbach TJ \& Mattes RD (2002) Effect of orosensory stimulation on postprandial thermogenesis in humans. Physiol Behav $\mathbf{7 5}$, $71-81$.

Vessby B, Tengblad S \& Lithell H (1994) Insulin sensitivity is related to the fatty acid composition of serum lipids and skeletal muscle phospholipids in 70-year-old men. Diabetologia 37, 1044-1050.

Woods SC (2004) Gastrointestinal satiety signals. I: An overview of gastrointestinal signals that influence food intake. Am J Physiol Gastrointest Liver Physiol 286, G7-G13. 